\title{
On the number of viewing zones required for head-tracked autostereoscopic display
}

\author{
Neil A. Dodgson \\ University of Cambridge Computer Laboratory, \\ 15 J J Thomson Avenue, Cambridge, UK, CB3 OFD
}

\begin{abstract}
A head-tracked display could be made from a two-view autostereoscopic display where head-tracking allows the display to swap the two views when the eyes move from viewing zone to viewing zone. Variations in human interpupillary distance mean that this basic two-view version will not work well for the significant minority of the population who have interpupillary distance significantly different from the average. Woodgate et al. proposed, in 1997, that a three-view system would work well. Analysis of an ideal version of their proposal shows that it does work well for the vast majority of the population. However, most multi-view, multi-lobe autostereoscopic displays have drawbacks which mean that, in practice, such a system would be unacceptable because of the inter-view dark zones generated by the inter-pixel dark zones on the underlying display technology. Variations of such displays have been developed which remove the inter-view dark zones by allowing adjacent views to overlap with one another: the views appear to smoothly blend from one to the next at the expense of a little blurring. Such displays need at least five viewing zones to accommodate the majority of the adult population with head-tracking and at least six viewing zones to accommodate everyone.
\end{abstract}

Keywords: stereoscopic, head-tracking, display, multi-view

\section{INTRODUCTION}

It has been suggested that a head-tracked display could be made from a two-view autostereoscopic display where the head-tracking allows the display to swap the two views when the eyes move from viewing zone to viewing zone. This paper analyses the number of views which are actually required for this type of system to work reliably. Variations in human interpupillary distance ${ }^{1}$ mean that the basic two-view version will not work well for the significant minority of the population who have interpupillary distance significantly different from the average. In particular, such a display designed for adults will be unsuitable for the majority of children. Woodgate et $a l^{2}$ postulate that a three-view system would work well. Analysis of an ideal version of their proposal shows that it does indeed work well for the vast majority of the population. However, most multi-view, multi-lobe autostereoscopic displays have drawbacks which mean that, in practice, such a system would not be acceptable because of the inter-view dark zones generated by the inter-pixel dark zones on the underlying liquid crystal or plasma display technology. This is certainly true of most lenticular, parallax barrier and parallax illumination displays. However, variations of these displays have been developed which remove the inter-view dark zones by allowing adjacent views to overlap with one another: the views appear to smoothly blend from one to the next at the expense of a little blurring. Analysis of these displays shows that it becomes necessary to have a least five viewing zones to accommodate the majority of the adult population with head-tracking and at least six viewing zones to accommodate everyone.

\section{TYPES OF AUTOSTEREOSCOPIC DISPLAY}

Autostereoscopic displays present multiple two-dimensional images on a single display surface. Each image is only visible in a limited zone in front of the display. There may be a single zone for each image or each image may be visible in multiple zones. In the latter case, the pattern of zones tends to repeat in multiple lobes, each lobe being a set of abutting zones in each of which a different image is visible. At one end of the autostereoscopic spectrum are the two-view displays, which either have exactly two viewing zones $^{3,4}$ or have multiple abutting

Copyright 2006 SPIE and IS\&T. This paper is published in Proc. SPIE vol. 6055 and is made available as an electronic preprint with permission of SPIE and IS\&T. One print or electronic copy may be made for personal use only. Systematic or multiple reproduction, distribution to multiple locations via electronic or other means, duplication of any material in this paper for a fee or for commercial purposes, or modification of the content of the paper are prohibited. 


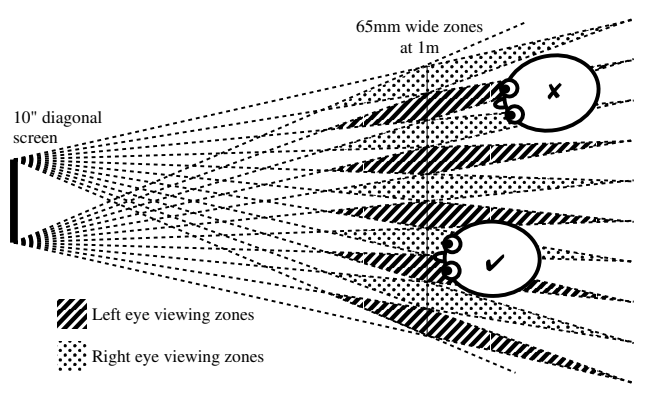

Figure 1. A two-view display produces multiple viewing zones, an eye in one of these zones will see either the left or the right image. An eye outside the shown zones will see an image made up of parts of both left and right images. Even at the ideal viewing distance there is a $50 \%$ chance that the viewer will see an incorrect, pseudoscopic image.

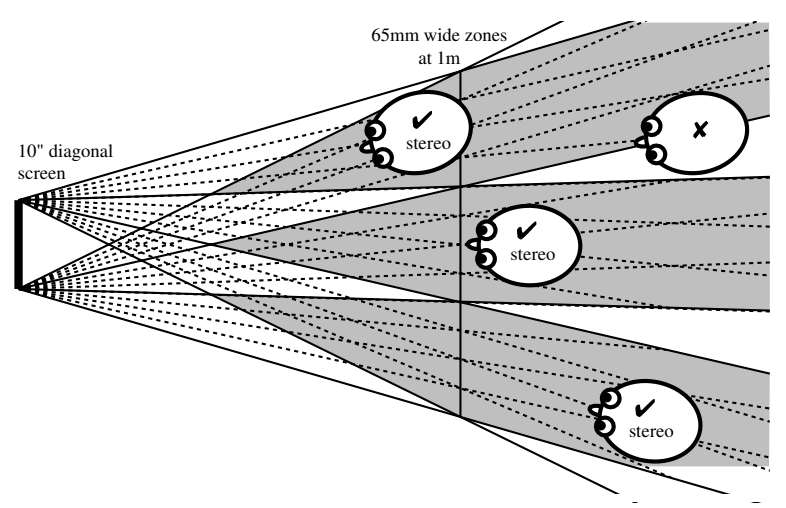

Figure 2. A four view autostereoscopic display with three lobes. Each of the lobes contains the same set of four views. So long as a viewer's head is within one of the lobes, a 3D image will be perceived.

lobes in which the two views repeat ${ }^{5,6}$ as shown in Figure 1. At the other end of the spectrum are the multi-view displays with a single lobe containing a large number of views. ${ }^{7}$

For any autostereoscopic display there will be an optimal distance from which to view the display. This is the distance at which all of the viewing zones abut, as can be seen in the examples in Figures 1 and 2 . Dodgson ${ }^{8,9}$ gives a full analysis of the viewing zone at the optimal distance and of what can be seen by the viewer at distances other than the optimum.

There is a huge range of methods for constructing an autostereoscopic display and such displays can be categorised in a wide variety of ways. ${ }^{10,11}$ One useful distinction is between those displays with two views and those with more. Another is between those displays which utilise head-tracking and those which do not. A third distinction is between those displays with a single lobe and those with multiple lobes. For example, Figure 1 shows a two view display with five lobes and Figure 2 shows a four view display with three lobes.

In this paper we consider two-view, multi-lobe, head-tracked displays and show that they are not practical. We then consider three- and more view, multi-lobe, head-tracked displays and derive results which show how useful these displays will be in practice. First, we consider some of the categories of display and the technologies used to implement them.

Send correspondence via e-mail to nad@cl.cam.ac.uk 


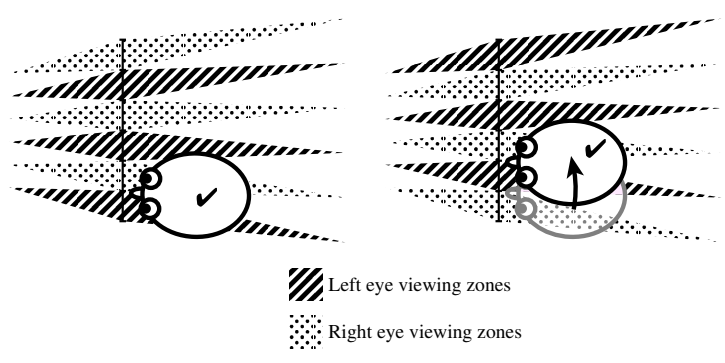

Figure 3. With head-tracking the zones can be swapped over as the viewer moves his or her head. This obviously only works for a single viewer at any one time.

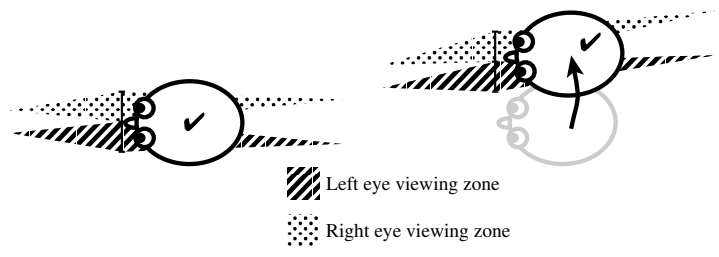

Figure 4. An alternative mechanism for head-tracking: only two zones are produced, but the image display mechanism can control where those two views are in space.

\subsection{Two-view displays}

It has long been known ${ }^{12}$ how to make a two-view autostereoscopic display using either parallax barrier ${ }^{13}$ or lenticular sheet ${ }^{14}$ technology. These divide, into two sets, the horizontal resolution of the underlying, typically liquid crystal, display device. One of the two visible images consists of every second column of pixels; the second image consists of the other columns. The two images are captured or generated so that one is appropriate for the viewer's left eye and one appropriate for the right. The two displayed images are visible in multiple lobes: each image is visible in several distinct zones in space (Figure 1). If the viewer stands at the optimal distance and in the correct position he or she will perceive a stereoscopic image. The downside of this is that there is a $50 \%$ chance of the viewer being in the wrong position and seeing an incorrect, pseudoscopic image. Moving much forward of or back from the optimal distance increases further the chance of seeing an incorrect image.

\subsection{Head-tracked displays}

Two-view technology is, as described above, well understood. If it were possible to know the position of the viewer's head then, in theory, the appropriate images, left and right, could be displayed to the appropriate zones, thus preventing any pseudoscopic viewing (Figure 3). This paper concerns itself with analysing this solution.

In practice, head-tracking with multi-lobe, two-view displays is usually achieved using some sort of mechanism for physically moving the viewing zones rather than just swapping the zones. For example, Eichenlaub's parallax illumination system ${ }^{5}$ has three sets of illumination bars, which can shift the viewing zones by one third of their width. In combination with the ability to switch left and right images between the two sets of zones, this makes a reasonable head-tracked display. Jewell et $a l^{15}$ describe a lenticular display where they move the lenticular sheet with respect to the LCD panel rather than switching images between zones.

There are a range of other technologies which display only two zones, and which allow these zones to be physically moved ${ }^{4,16}$ (Figure 4 ).

There are three disadvantages to head-tracking displays methods. The first is that the head-tracking requires some mechanism in addition to the display itself. The head-tracking mechanism must not require the user to wear anything special: it would be pointless to replace the wearing of special glasses with the wearing of a special 
head-tracker. While this has been a difficult technical problem, head-tracking systems are now sophisticated enough to make them practical for use with autostereoscopic displays. ${ }^{2}$

The second disadvantage of many head-tracking displays is that they require some component to be moved physically. This carries the problem that mechanical components require significantly more maintenance than electronic components. The advantage of the head-tracked systems investigated in this paper is that there are no moving parts.

The other limitation of most head-tracked systems is that they are single-viewer. This is acceptable in some applications but not in others.

\subsection{Multi-view displays}

Multi-view displays display multiple different images to multiple zones in space as illustrated in, for example, Figure 2. This has the advantages that the viewer is free to place his or her head anywhere within the viewing lobe, while still perceiving a 3D image; the viewer can 'look around' objects in the scene simply by moving his or her head; multiple viewers can be supported, each seeing 3D from his or her own point of view; and head-tracking is not required.

The disadvantages of multi-view displays are: the difficulty of building a display with many views; the problem of generating all the views simultaneously, ${ }^{17}$ because each view is being displayed all of the time, whether anyone can see that particular view or not; and the fact that, for a given underlying display mechanism, a two-view or head-tracked display will always have higher resolution than a multi-view display because the number of pixels displayed have to be shared across fewer views than in a multi-view system.

In this paper, we consider combining head-tracking with a multi-view display which has only a small number of views (3-6), these views visible in multiple abutting lobes. The advantage of such displays is that they are relatively easy to construct, have a higher resolution than the equivalent multi-view display with a larger number of views, and they are inexpensive compared to many other autostereoscopic technologies. Their disadvantage is that their low number of views makes them practically unusable without head-tracking.

These displays are usually based on LCD panels. Some optical mechanism is added to the panel to produce multiple images, each visible in its own set of zones. There are four principal optical mechanisms by which this can be achieved: lenticular lenslets, ${ }^{6,18}$ parallax barrier, ${ }^{6}$ parallax illumination, ${ }^{5}$ and holographic optical elements. $^{3}$ These all subdivide the resolution of the underlying LCD panel into multiple images. So, for a given LCD panel, the smaller the number of independent views, the higher the resolution in each view's image.

\section{VARIATIONS IN INTERPUPILLARY DISTANCE}

In order to carry out any sensible analysis, we need to know the extent of variations in human interpupillary distance (IPD). Mean IPD is an important and often quoted statistic required in the design of stereoscopic and autostereoscopic viewing systems. In this work we are interested in not just the mean IPD but also the extrema. Dodgson $^{1}$ summarises the variations in IPD:

Mean IPD depends on the characteristics of the population from which the data is drawn. Mean IPD is statistically significantly different between the two genders, between certain racial groups, between near and far viewing, and between certain age groups. Mean and median IPD for the adult human population both appear to lie somewhere near $63 \mathrm{~mm}$. With regard to extrema: the vast majority of adults lie within the range 50 to $75 \mathrm{~mm}$. There are several cases of people outside this range so, to ensure that all adults are accommodated, I recommend a range of 45 to $80 \mathrm{~mm}$, although there is at least one known case ${ }^{19,20}$ of a 15 year old female with an IPD of $43 \mathrm{~mm}$, which is outside even this extended range. If you wish to take into account children down to age five, then the minimum should be reduced to $40 \mathrm{~mm}$. In the (unlikely) event that you need to consider newborn babies, the minimum should be reduced still further, perhaps as low as $30 \mathrm{~mm}$.

An autostereoscopic display should thus be able to cope with IPDs of 50-75 $\mathrm{mm}$ for the majority adult viewers and 40-80 $\mathrm{mm}$ to cope with everyone, including children down to age five. 


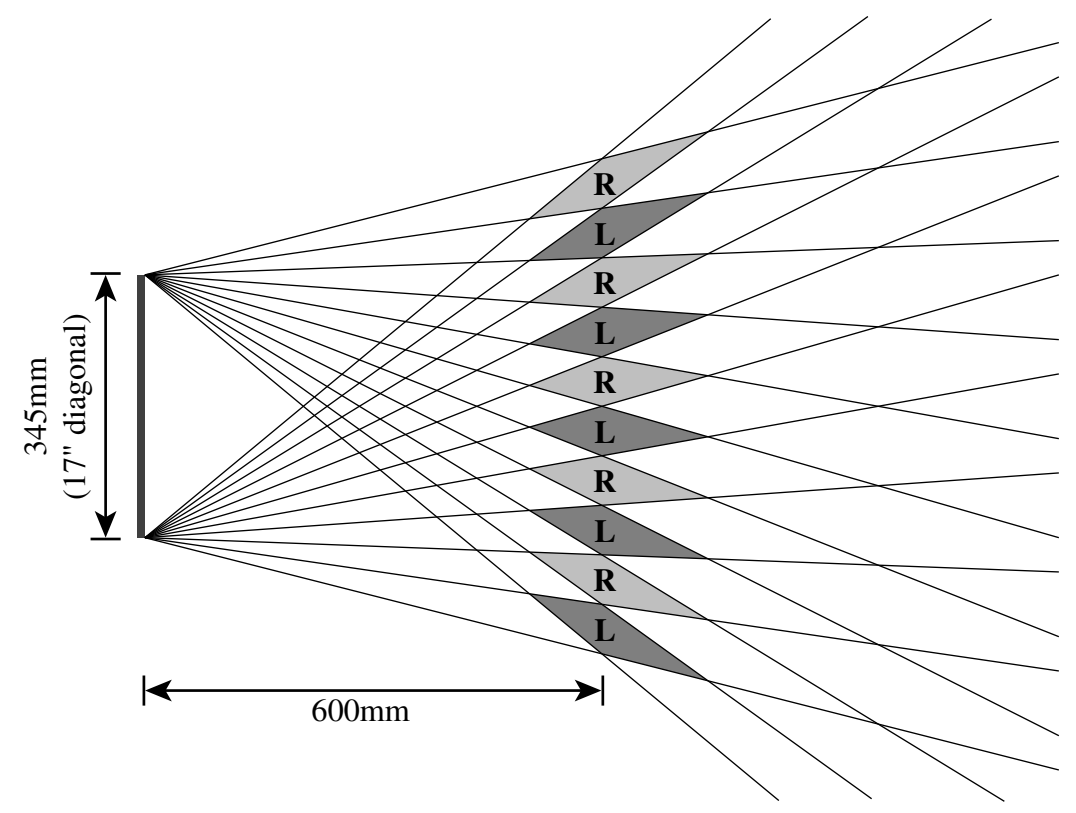

Figure 5. The viewing zones of a 17" diagonal two-view display with an optimal distance of $600 \mathrm{~mm}$. At the optimal distance, the viewing zones are $65 \mathrm{~mm}$ wide, close to mean human IPD. For correct stereoscopic viewing, the viewer must place their left eye in a left-eye viewing zone (labelled $\mathbf{L}$ ), and their right eye in a right-eye viewing zone (labelled $\mathbf{R}$ ).

\section{IMPLICATIONS FOR HEAD-TRACKED TWO-VIEW AUTOSTEREOSCOPIC DISPLAYS}

Consider an ideal two-view, multi-lobe autostereoscopic display without head-tracking. In order to see a correct stereoscopic image, the viewer must have his left eye in a left-eye viewing zone and his right eye in a right-eye viewing zone. These zones are illustrated in Figure 5.

In the following analysis, we assume that the display has been constructed for a human with IPD of $65 \mathrm{~mm}$ (this is mean IPD rounded to the nearest $5 \mathrm{~mm}$ and coincidentally corresponds to Hofstetter's adult, white, American male $\left.{ }^{1,21}\right)$. We illustrate correct viewing locations by showing where the viewer must place his or her left eye. First, consider a person with the same IPD as that for which the display has been designed. He must place his left eye in one of the zones shown in Figure 6(b). These zones in Figure 6(b) obviously correspond to the left-eye viewing zones in Figure 5 (repeated for convenience in Figure 6(a)). This viewer has limited head movement: at the widest point he can move his head left-right a distance equal to the designed viewing zone separation of $65 \mathrm{~mm}$. As this is not a very large distance, he must keep his head practically stationary in order to maintain stereoscopic perception.

Now consider a person with IPD significantly less than the display's designed viewing zone separation. At the optimal distance she has the possibility of having both eyes in the same viewing zone - providing a monoscopic rather than stereoscopic image. She thus has smaller valid viewing zone than a viewer with IPD equal to the designed viewing zone separation, as shown in Figure 6(c), and consequently less available head movement. By a similar analysis, a person with IPD significantly larger than the designed viewing zone separation also has a reduced range of valid positions.

Now, add head-tracking to such a two-view display. Assume perfect head-tracking so that the display is always displaying the left view to the zone in which the left eye is present, if the left eye is at the optimal distance. Away from the optimal distance, the correct zone is chosen by drawing a ray from the centre of the screen, passing through the left eye. Whichever zone this passes through at the optimal distance is the correct zone in which to display the left view. 


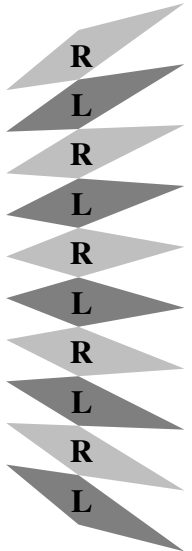

(a)

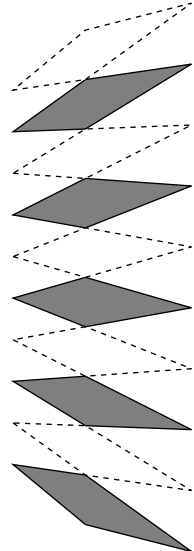

(b)

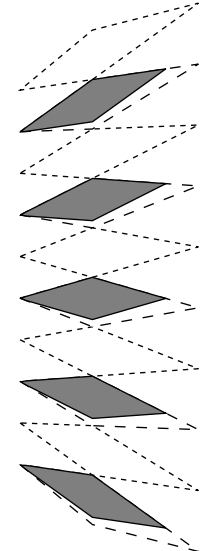

(c)

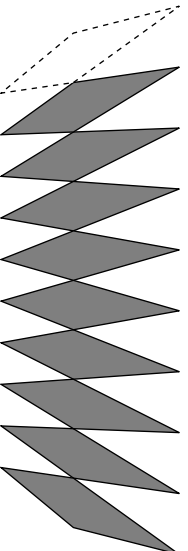

(d)

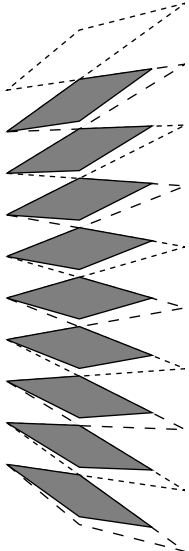

(e)

Figure 6. Positions of the left eye which will result in a correct stereoscopic image. (a) shows the viewing zones for left and right eye from Figure 5. (b)-(e) show, shaded in grey, valid positions of the left eye for correct stereoscopic viewing. (b) for viewer with IPD equal to that for which the display has been designed, in this case it is set to be $65 \mathrm{~mm}$. (c) for a viewer with IPD significantly less than that for which the display has been designed, in this case $55 \mathrm{~mm}$. (d) with head-tracking for a $65 \mathrm{~mm}$ IPD. (e) with head-tracking for a $55 \mathrm{~mm}$ IPD.

For a viewer with the display's designed IPD, the valid positions of the left eye are now as shown in Figure 6(d). As can be seen, at the optimal distance, the viewer has freedom to move his head left-right and should see no pseudoscopic artifacts at any point, so long as he stays at the optimal distance. However, a viewer with IPD greater than or less than that for which the display is designed will have significant areas of monoscopic viewing at the optimal distance, as illustrated in Figure 6(e). The amount of error can be quantified. Let the views be set to be $65 \mathrm{~mm}$ wide. For an IPD of $55 \mathrm{~mm}$, the proportion of locations at the optimal distance at which the viewer will see a monoscopic, rather than a stereoscopic, image can be calculated as $1-\frac{55}{65} \approx 17 \%$. The same percentage holds for a larger than designed IPD of $75 \mathrm{~mm}$. Regardless of IPD, a viewer positioned much forward of or back from the optimal distance will see an incorrect "mosaiced" image where one or both eyes sees an image made up of parts of both the left and the right views.

We can therefore conclude that a two-view head-tracked autostereoscopic display designed for a viewer with mean IPD will be unsuitable for the significant minority of the population who have IPD significantly different to the mean. In addition, a display designed for adults will be unsuitable for the majority of children. Furthermore, even for those viewers with IPD close to the mean, there is a requirement that they sit at or very close to the optimal distance to ensure that they see correct imagery at all positions as they move their heads left-right.

\section{THE IDEAL THREE VIEW SYSTEM}

Woodgate et al postulate that a solution to this problem is to use three views. ${ }^{2}$ In this section we provide a theoretical analysis of this system for an ideal three-view head-tracked autostereoscopic display. Subsequent sections consider the additional problems introduced by using a real autostereoscopic display. Figure 7 shows how such a display is configured. There are three independent images, each visible in a set of zones arranged so that the zones abut at the optimal distance. Each of the viewing zones has a width two-thirds of mean IPD.

For our purposes, we have two images: one for the left eye and one for the right eye. At any given time, two of the three views are showing the image for one eye while the other view shows the image for the other eye (Figure 8). The choice of whether to show the left image or the right image in a particular view is taken by the location of the two eyes. The views in which the two eyes are present must obviously show the left and right images respectively. The third view will show the image for whichever eye is closest to being in that view, remembering that the views repeat multiple times across the whole viewing zone. 


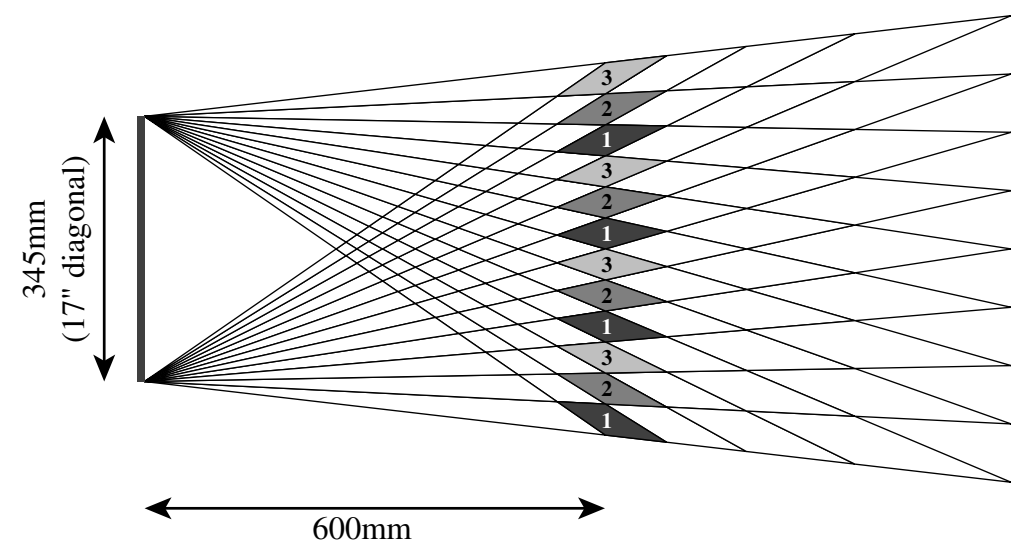

Figure 7. A three-view, multi-lobe display, where the viewing zones for each view are indicated by the appropriate numbers.

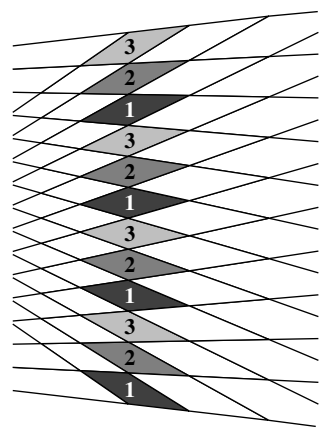

(a)

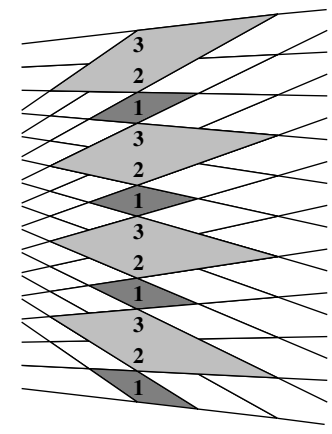

(b)

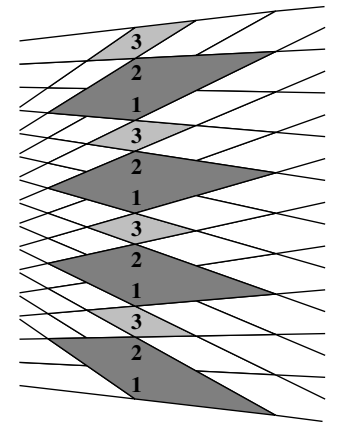

(c)

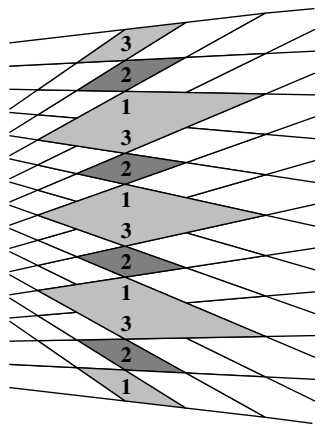

(d)

Figure 8. Using a three-view display for head-tracked autostereoscopic viewing. (a) shows the viewing zones from Figure 7. (b) shows the left (dark) and right (light) viewing zones when the left image is displayed in view 1 and the right in views 2 and 3. The viewer's left eye must be in the left eye viewing zone (zone 1) and the viewer's right eye in the right eye viewing zone. Note that displaying the same image in both of views 2 and 3 dramatically increases the size of this viewing zone. (c) and (d) show two other configurations with the same image displayed in views 1 and 2 and in views 3 and 1 respectively.

Let $\beta$ be the width of the viewing zone. Consider a viewer at the optimal distance. It is vital that both eyes are in different zones to prevent monoscopic viewing. IPD must therefore be at least $\beta$ (to prevent eyes from ever being in the same zone) and at most $2 \beta$ (to prevent eyes from being in zones three apart which, by the nature of the display, always show the same image). Setting $\beta=40 \mathrm{~mm}$ gives a display which can accommodate the full range of human IPDs, from the narrowest IPD of a five year-old to the widest IPD of an adult. All viewers will see valid stereoscopic images at the optimal distance, thus addressing the first of the two problems in section 4.

The second problem, that of allowing for fore-back head movement, also needs to be analysed. Consider a person viewing the display configured as in Figure 8(b). His left eye is in zone 1, while his right eye is in the larger zone created by displaying the same image to views 2 and 3 . Now, let the viewer move his head to the right. At the point where his left eye leaves zone 1, zone 2 must switch (or have already switched) from displaying the right eye's image to displaying the left eye's image (Figure 8(c)). This means that the right eye must have entered zone 3 by this point. This provides a hard limit on the minimum IPD which will allow correct stereoscopic viewing. This minimum IPD is the distance from the right-hand edge of zone 1 to the left-hand edge of zone 3 (Figure $9(\mathrm{a})$ ).

By a similar argument, we can find a hard upper limit on IPD by considering what happens as the left 


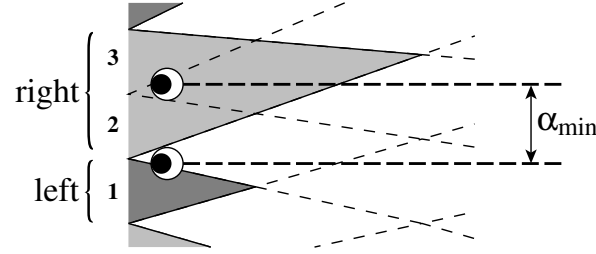

(a)

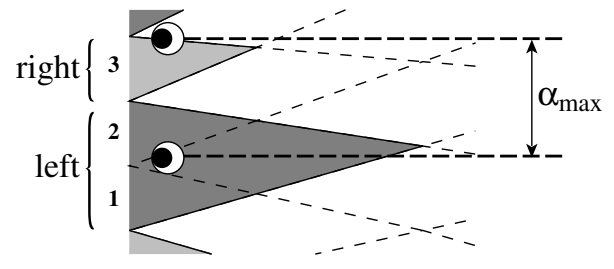

(b)

Figure 9. (a) The minimum IPD is the distance from the right hand edge of one zone (zone 1 in this example) to the left hand edge of the zone two to its right (zone 3 in this example). (b) The maximum IPD is the distance from the left hand edge of one zone (zone 2 in this example) to the right hand edge of the zone immediately to its right (zone 3 in this example).

eye continues to move further to the right. At some point it enters zone 2 and this allows zone 1 to switch to displaying the right eye's image (Figure $8(\mathrm{~d})$ ) and therefore gives the right eye the freedom to move out of zone 3. The maximum IPD is thus the distance from the left-hand age of zone 2 to the right-hand edge of zone 3 (Figure 9(b)).

From these we can derive for equations determining the maximum $\left(\alpha_{\max }\right)$ and minimum $\left(\alpha_{\min }\right)$ IPD for any distance forward of $\left(\delta_{\text {front }}\right)$ or back from $\left(\delta_{\text {back }}\right)$ the optimal distance:

$$
\begin{aligned}
\text { Front: } \alpha_{\min } & =\beta+\frac{\delta_{\text {front }}}{d_{o}}\left(w_{l}-\beta\right) \\
\alpha_{\max } & =2 \beta-\frac{\delta_{\text {front }}}{d_{o}}\left(w_{l}+2 \beta\right) \\
\text { Back: } \alpha_{\min } & =\beta+\frac{\delta_{\text {back }}}{d_{o}}\left(w_{l}+\beta\right) \\
\alpha_{\max } & =2 \beta-\frac{\delta_{\text {back }}}{d_{o}}\left(w_{l}-2 \beta\right)
\end{aligned}
$$

Alternatively, for a given IPD, $\alpha$, we can determine the maximum available movement forward of and back from the optimal distance, and therefore the maximum total movement, $\delta=\delta_{\text {front }}+\delta_{\text {back }}$.

$$
\begin{aligned}
\delta_{\text {front }} & =\min \left((\alpha-\beta) \frac{d_{o}}{w_{l}-\beta},(2 \beta-\alpha) \frac{d_{o}}{w_{l}+2 \beta}\right) \\
\delta_{\text {back }} & =\min \left((\alpha-\beta) \frac{d_{o}}{w_{l}+\beta},(2 \beta-\alpha) \frac{d_{o}}{w_{l}-2 \beta}\right)
\end{aligned}
$$

In Figure 10 we graph these values for our example display, viewing zone width $\beta=40$ mm, screen width $w_{l}=345 \mathrm{~mm}$, and optimal viewing distance $d_{o}=600 \mathrm{~mm}$. The maximum fore-back freedom of movement is roughly $10 \%$ of the optimal distance for viewers with an IPD of 57-64 mm. Thus an ideal, three-view, multi-lobe, head-tracked autostereoscopic display provides a good stereoscopic effect for all IPDs at the optimal distance and a significant range of fore-back movement for the vast majority of the human population.

\section{LIMITATIONS OF PRACTICAL DISPLAYS}

The above analysis is valid for the ideal three-view, multi-lobe autostereoscopic display and for any practical

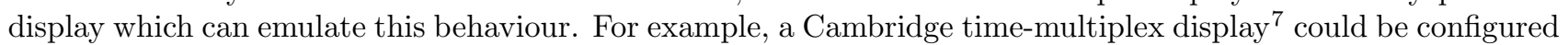
to behave in this way.

However, the majority of practical autostereoscopic display technologies suffer from drawbacks which means that the above analysis must be modified. In this section we consider the four principal optical mechanisms which can be applied to a liquid crystal or plasma display panel to produce an autostereoscopic display. Two-view 


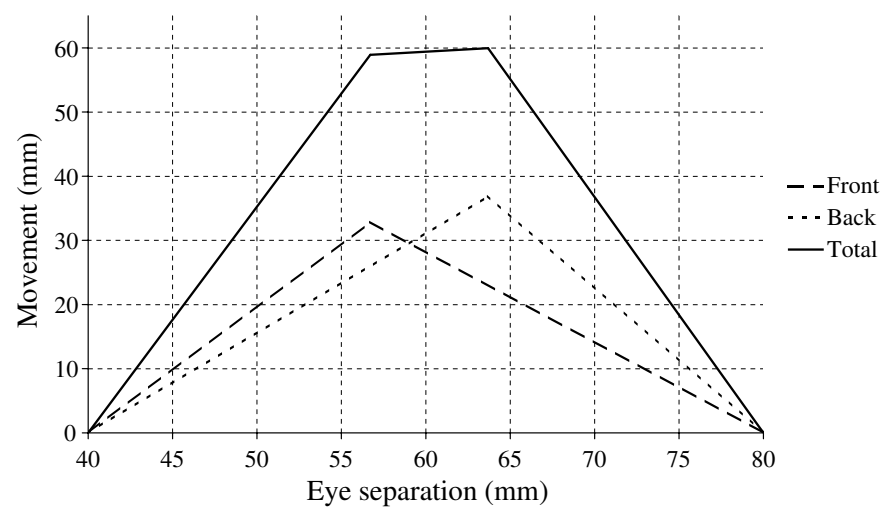

Figure 10. Maximum forward (Front) and backward (Back) movement from the optimal distance for an ideal three-view, multi-lobe, head-tracked display with parameters: $\beta=40 \mathrm{~mm}, w_{l}=345 \mathrm{~mm}, d_{o}=600 \mathrm{~mm}$. Total is the sum of Front and Back. This graph is also correct for the equivalent six-view display with $100 \%$ overlap between viewing zones and $\beta=20 \mathrm{~mm}$ (Section 7).

displays have been made using all four technologies and it would be straightforward to make a three-view display using any of the four.

\subsection{Lenticular sheets}

Each lenslet on a lenticular sheet projects the rows of pixels out so that, as the optimal distance, a pixel is magnified to be the width of a viewing zone. The problem with lenticular sheets is that they also magnify the inter-pixel gaps. This means that there are dark zones between the views in which no image is visible on screen at the optimal distance. Therefore, for a two-view display, even a person with the ideal IPD will have invalid positions at the optimal distance. At other distances these dark zones manifest themselves as moiré-like fringes which appear to run across the screen when the viewer move his head from side to side. ${ }^{18}$ Because of this effect, the above three-view solution will not work.

Woodgate et $a .^{2}$ addressed the problem by designing a LCD panel with a novel pixel layout, which they refer to as PIXCON. The distributes the pixels evenly so as to ameliorate these artifacts.

However, most LCD panels do not benefit from the PIXCON layout and alternative solutions must be sought. van Berkel ${ }^{18}$ and Lipton ${ }^{22}$ both describe lenticular displays which avoid the dark zone problem by slanting the lenticular array with respect to a conventional LCD pixel array. While this removes the dark zones, it also has the effect of blurring the boundaries between views. Indeed, in both of the displays described by these authors, the overlap between views is $100 \%$. Thus, for example, view 3 is only visible as an image in its own right from exactly one location at the optimal distance. As the viewer moves his eye to the left, view 3 blends smoothly into view 2 and, as the viewer moves his eye to the right, it blends smoothly into view 4 . This will not allow the three-view solution described above. To address this problem, which is also faced by parallax barrier and parallax illumination systems, Section 7 (below) extends the above analysis to displays with $100 \%$ overlap between views and shows that at least five views are required.

\subsection{Parallax barrier and parallax illumination}

These two display types work in similar ways. They use an array of optical apertures each of which is aligned with at least two columns of the LCD pixels. Such displays have been demonstrated with both front or rear positioning of the apertures. The slits may be embodied as apertures in a transmission mask (parallax barrier) or as imaged light lines (parallax illumination).

To produce sufficient light output there needs to be a region of overlap between adjacent views. The proportion of one view which overlaps with an adjacent view is $w_{s} / w_{p}$, where $w_{s}$ is the width of the slit (parallax barrier) or illumination bar (parallax illumination) and $w_{p}$ is the pixel width (that is: the distance from the left-hand edge 
of one pixel to the left-hand edge of the next pixel to its right). For zero overlap, these displays work in a similar manner to lenticular displays: they project the individual pixels to the viewing zones. This means that they also suffer from the dark band effect as the inter-pixel gaps are also projected out. However, to produce sufficient light output in a parallax barrier display, $w_{s}$ must be a significant fraction of $w_{p}$, which significantly reduces the dark band effect at the expense of overlap between views. The ramifications of this overlap are discussed in Section 7 (below).

\subsection{Holographic optical elements}

Displays which use holographic optical elements can be manufactured so that the views abut at the optimal distance. There is thus no dark band effect and no overlap. A three-view holographic optical element display can thus be built to work as described above.

\section{ANALYSIS OF DISPLAYS WITH OVERLAP}

Conventional lenticular, parallax barrier, and parallax illumination autostereoscopic displays all project the interpixel dark zones out to be inter-view dark zones at the optimal distance. These dark zones manifest as regions in which the screen goes completely dark (this will only occur at or very close to the optimal distance) or as dark bands which run across the screen as the viewer moves his head. By allowing the views to overlap with one another, as described above, these artifacts can be ameliorated. However, unless overlap is 100\%, the artifacts will be visible to a greater (low or zero overlap) or lesser (high overlap) extent. This essentially means that the above three-view solution, while accurate for an ideal display, is inappropriate for these displays. In this section, we extend our analysis by considering what behaviour is exhibited by displays with $100 \%$ overlap.

Let $\beta_{n}$ be the viewing zone width at the optimal distance for a display with $n$ views. Analysis of the different cases, similar to that given above, determines hard limits on the range of human IPD $(\alpha)$ which can be accommodated at the optimal distance by differing numbers of views for a display with $100 \%$ overlap:

$$
2 \beta_{n} \leq \alpha \leq(n-2) \beta_{n}
$$

Figure 11 gives a pictorial analysis of the situation in a form similar to that in Figures 8 and 9. There are always two zones which contain a blend between the left eye's image and the right eye's image. It is important that neither eye is ever in one of these zones. The remainder of the zones are divided between one or more abutting zones in which only the left eye's image is visible and one or more abutting zones in which only the right eye's image is visible.

Now, consider how many views are required to accommodate a reasonable range of human IPDs. A four-view display is obviously unusable, as the upper and lower limits on IPD are equal. This is the equivalent of a two view display without overlap (Section 4).

A five-view display will work for the majority of the adult population. For example, a view zone width of $25 \mathrm{~mm}$ would allow the range of IPDs 50-75 $\mathrm{mm}$.

A six-view display with a view zone width of $20 \mathrm{~mm}$ would allow the range of IPDs $40-80 \mathrm{~mm}$, matching that of the ideal three-view display.

We can again produce formulae, giving the maximum fore and back movement for any number of viewing zones.

$$
\begin{aligned}
& \delta_{\text {front }}=\min \left((\alpha-2 \beta) \frac{d_{o}}{w_{l}-2 \beta},((n-2) \beta-\alpha) \frac{d_{o}}{w_{l}+(n-2) \beta}\right) \\
& \delta_{\text {back }}=\min \left((\alpha-2 \beta) \frac{d_{o}}{w_{l}+2 \beta},((n-2) \beta-\alpha) \frac{d_{o}}{w_{l}-(n-2) \beta}\right)
\end{aligned}
$$

Note that the six-view $100 \%$ overlap display has the same characteristics as the three-view $0 \%$ overlap display. In particular, Figure 10 is correct for the six-view 100\% overlap display. 


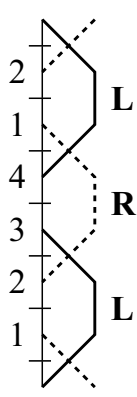

(a)

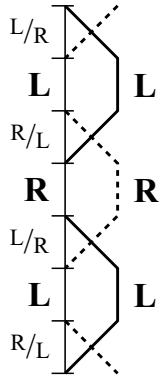

(b)

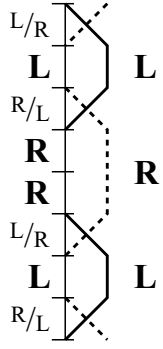

(c)

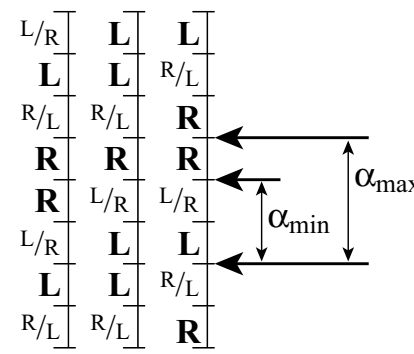

(i) (ii) (iii)

(d)

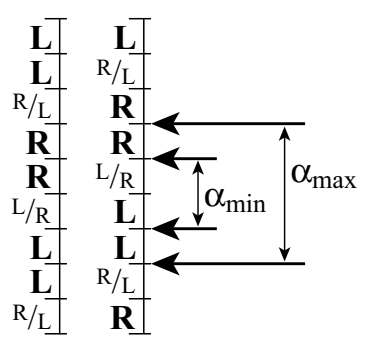

(i) (ii)

(e)

Figure 11. (a) illustrates what happens at the optimal distance for a four view display with $100 \%$ overlap between views. Views 1 and 2 are displaying the left eye' image $(\mathbf{L})$, while views 3 and 4 are displaying the right eye' image $(\mathbf{R})$. The overlap region between left and right thus starts half way across view 2 and ends half way across view 3 . A similar, one-view wide overlap region exists for the overlap from right to left between views 4 and 1. (b) shows the same situation, this time the one-view wide regions are labelled as left or right, or as a transition region. Note that these regions are offset by half a viewing zone width from the actual viewing zones. (c) gives the same diagram for a five-view display with $100 \%$ overlap. Note that two regions are now labelled as showing just the right eye image. (d) shows three configurations of the five-view display. The display would switch from (i) to (ii) to (iii) as the head is moved to the right. (i) and (ii) determine the minimum possible IPD at the optimal distance $\left(\alpha_{\min }\right)$ while (ii) and (iii) determine the maximum $(\alpha \max )$. (e) shows the same thing for a six-view display.

\section{SUMMARY}

A two-view autostereoscopic display with head-tracking is not practically usable. A significant minority of people have interpupillary distance so different from the mean that they would be seriously inconvenienced. In addition, the remainder of the population would need to sit very close to the optimal distance to avoid inconvenient effects.

By contrast, a three-view display with head-tracking works well for everyone, provided that the three view display has viewing zones which each display just one view.

For displays with blended overlap between adjacent viewing zones, a four-view display has the same problems as a two-view display without overlap. Five views are needed to ensure that head-tracking works well for the majority of people and six views for it to work for everyone.

\section{Acknowledgements}

Thanks to the Computer Science Department at the University of North Carolina at Chapel Hill and the School of Mathematical and Computing Sciences at the Victoria University of Wellington for their hospitality and use of their facilities during the preparation of this paper.

\section{REFERENCES}

1. N. A. Dodgson, "Variation and extrema of human interpupillary distance," in Stereoscopic Displays and Virtual Reality Systems XI, A. J. Woods, J. O. Merritt, S. A. Benton, and M. T. Bolas, eds., Proc. SPIE 5291, pp. 36-46, 2004.

2. G. J. Woodgate, D. Ezra, J. Harrold, N. S. Holliman, G. R. Jones, and R. R. Moseley, "Observer tracking autostereoscopic 3D display systems," in Stereoscopic Displays and Virtual Reality Systems IV, S. S. Fisher, J. O. Merritt, and M. T. Bolas, eds., Proc. SPIE 3012, pp. 187-198, 1997.

3. D. Trayner and E. Orr, "Autostereoscopic display using holographic optical elements," in Stereoscopic Displays and Virtual Reality Systems III, S. S. Fisher, J. O. Merritt, and M. T. Bolas, eds., Proc SPIE 2653, pp. 65-74, 1996. 
4. P. Harman, "Autostereoscopic display system," in Stereoscopic Displays and Virtual Reality Systems III, S. S. Fisher, J. O. Merritt, and M. T. Bolas, eds., Proc SPIE 2653, pp. 56-64, 1996.

5. J. B. Eichenlaub, "An autostereoscopic display with high brightness and power efficiency," in Stereoscopic Displays and Virtual Reality Systems, S. S. Fisher, J. O. Merritt, and M. T. Bolas, eds., Proc. SPIE 2177, pp. 4-15, 1994.

6. T. Okosi, Three-Dimensional Imaging Techniques, Academic Press, 1976.

7. N. A. Dodgson, J. R. Moore, S. R. Lang, G. Martin, and P. Canepa, "Time-sequential multi-projector autostereoscopic 3D display," J. Soc. for Information Display 8(2), pp. 169-176, 2000.

8. N. A. Dodgson, "Analysis of the viewing zone of the Cambridge autostereoscopic display," Applied Optics: Optical Technology \&3 Biomedical Optics 35(10), pp. 1705-1710, 1996.

9. N. A. Dodgson, "Analysis of the viewing zone of multi-view autostereoscopic displays," in Stereoscopic Displays and Virtual Reality Systems IX, Proc. SPIE 4660, pp. 254-265, 2002.

10. N. A. Dodgson, "Autostereoscopic 3D displays," Computer 38(8), pp. 31-36, 2005.

11. N. A. Dodgson, J. R. Moore, and S. R. Lang, "Multi-view autostereoscopic 3D display," in International Broadcasting Convention, IBC 99, pp. 497-502, (10-14 Sept., Amsterdam), 1999. ISBN 0-9533673-1-2.

12. M. Starks, "Stereoscopic video and the quest for virtual reality: an annotated bibliography of selected topics - part II," in Stereoscopic Displays and Applications III, J. O. Merritt and S. S. Fisher, eds., Proc. SPIE 1669, pp. 216-227, 1992.

13. J. B. Eichenlaub, "Developments in autostereoscopic technology at Dimension Technologies Inc.," in Stereoscopic Displays and Applications IV, J. O. Merritt and S. S. Fisher, eds., Proc. SPIE 1915, pp. 177-186, 1993.

14. D. F. McAllister, ed., Stereo Computer Graphics and Other True 3D Technologies, Princeton University Press, Princeton, New Jersey, 1993. ISBN 0-691-08741-5.

15. M. R. Jewell, G. R. Chamberlin, D. E. Sheat, P. Cochrane, and D. J. McCartney, "3-D imaging systems for video communication applications," in Stereoscopic Displays and Virtual Reality Systems II, S. S. Fisher, J. O. Merritt, and M. T. Bolas, eds., Proc. SPIE 2409, pp. 4-10, 1995.

16. D. Ezra, G. J. Woodgate, B. A. Omar, N. S. Holliman, J. Harrold, and L. S. Shapiro, "New autostereoscopic display system," in Stereoscopic Displays and Virtual Reality Systems II, S. S. Fisher, J. O. Merritt, and M. T. Bolas, eds., Proc. SPIE 2409, pp. 31-40, 1995.

17. O. M. Castle, "Synthetic image generation for a multiple-view autostero display," Tech. Rep. 382, University of Cambridge Computer Laboratory, 15 J. J. Thompson Avenue, Cambridge, UK CB3 0FD, Oct 1995. ISSN 1476-2986.

18. C. van Berkel and J. A. Clarke, "Characterisation and optimisation of 3D-LCD module design," in Stereoscopic Displays and Virtual Reality Systems IV, S. S. Fisher, J. O. Merritt, and M. T. Bolas, eds., Proc. SPIE 3012, pp. 179-186, 1997.

19. C. Evereklioğlu, S. Doğanay, H. Er, and A. Gündüz, "Distant and near interpupillary distance in 3448 male and female subjects: final results," Turgut Özal Tip Merkezi Dergisi 6(2), pp. 84-91, 1999.

20. H. B. Pryor, "Objective measurements of interpupillary distance," Pediatrics 44, pp. 973-977, 1969.

21. H. W. Hofstetter J. Am. Optometric Assoc. 43, pp. 1151-1155, 1972.

22. L. Lipton and M. Feldman, "A new autostereoscopic display technology: The SynthaGram ${ }^{\mathrm{TM}}$," in Stereoscopic Displays and Virtual Reality Systems IX, Proc. SPIE 4660, pp. 229-235, 2002. 